\title{
ASTMH-Approved Diploma Courses in Clinical Tropical Medicine
}

\author{
Baylor College of Medicine \\ Bernhard Nocht Institute \\ Case Western Reserve University (through 2003) \\ Gorgas Memorial Institute, University of Alabama \\ Humboldt University \\ Johns Hopkins University \\ Liverpool School of Tropical Medicine
}

London School of Hygiene and Tropical Medicine

Mahidol University

Tulane University

Uniformed Services University of the Health Sciences

University of Virginia

West Virginia University 